\title{
Probable Entropic Nature of Gravity in Ultraviolet and Infrared Limits_-Part I: An Ultraviolet Case
}

\author{
A. E. Shalyt-Margolin \\ National Centre of Particles and High Energy Physics, Bogdanovich Street 153, 220040 Minsk, Belarus \\ Correspondence should be addressed to A. E. Shalyt-Margolin; a.shalyt@mail.ru
}

Received 2 May 2013; Accepted 21 August 2013

Academic Editor: Shi-Hai Dong

Copyright (C) 2013 A. E. Shalyt-Margolin. This is an open access article distributed under the Creative Commons Attribution License, which permits unrestricted use, distribution, and reproduction in any medium, provided the original work is properly cited.

This work presents a study of the possibility for extending the well-known results of E. Verlinde concerning the entropic nature of gravity to the ultraviolet region (Planck's energies) and also the derivation of quantum corrections to Einstein Equations.

\section{Introduction}

In the last 15-20 years, new and very interesting approaches to gravity studies have been proposed, which may be divided into "thermodynamical" and "theoretical informational" approaches. The approach suggested in the pioneer work by Jacobson [1] has been considerably extended in a series of remarkable papers by Padmanabhan [2-13]. The paper by Verlinde [14] stating a secondary character of the gravitational interaction and its entropic nature was published in 2010 after the appearance in the ArXiv. The paper [14] introducing such specific terms as "entropic force" has been followed by numerous studies (e.g., [15-26] and others).

In this work, the author studies the possibility for extension of the results given in [14] to the ultraviolet region (Planck's energies) and presents the derivation of quantum corrections to Einstein Equations using the dimensionless small parameter $\alpha$ introduced by the author in his previous works [27-39].

\section{Fundamental Quantities and Their High-Energy Deformation}

In this section, the author uses the "ideology," terms, and notation introduced in [14] to extend the corresponding results to the ultraviolet and infrared gravity regions. It may be stated that the results in [14] have been obtained for the "medium" energies, that is, for the range of well-known energies, where the General Relativity (GR) is valid. But owing to modern knowledge, in the ultraviolet and infrared limits, gravity may be modified. As regards to the ultraviolet (Planck) scale, this idea has been proposed long ago [40-45], and in this situation we have to replace the word "may" by the word "must". As for low energies, there are many recent publications considering the infrared (for great distances) modification of gravity (e.g., [46, 47]). And the modification can have a solid experimental status in the nearest future [48].

Naturally, when we are concerned with extension of some results to higher or lower energies, the principle of conformity must be executed undeviatingly; ongoing to the known energy scales, the known results must be reproduced.

In this section, it is shown that the fundamental quantities $A, N$, and $T$, defined in [14] and associated with the holographic screen (where $A$-its surface area, $N$-number of data bits "existing" on $A, T$-its temperature), may be supplementary defined for the region of high energies so that at normal energies they be coincident with values given in [14]. In the process, we take the corresponding quantities for the stationary Schwarzschild black hole as the most natural holographic object.

The idea is as follows: formulae for the correction of the fundamental quantities within the Generalized Uncertainty Principle (GUP) for the holographic screen $\mathcal{S}$ from [14] are similar to those for the black hole. 
Let us consider Section 5.2 (Derivation of Einstein Equations) in [14]. In this section, formula (5.32) for a "bit density" on the holographic screen is given as

$$
d N=\frac{d A}{G \hbar} .
$$

However, when the holographic principle [49-53] is valid, $N$ is actually the entropy $S$ up to the factor $S \sim N$, and hence from (1) it follows directly that

$$
d S \sim \frac{d A}{G \hbar} .
$$

What are the changes in $S$ ongoing to high (Planck) energies? The answer to this question is already known owing to the fact that at these energies the Heisenberg Uncertainty Principle (HUP) is replaced by GUP [54-61]:

$$
\Delta x \geq \frac{\hbar}{\Delta p}+\ell^{2} \frac{\Delta p}{\hbar}
$$

where $\ell^{2}=\alpha^{\prime} l_{p}^{2}$ and $\alpha^{\prime}$ is the dimensionless numerical factor. The well-known Bekenstein-Hawking formula for the black hole entropy in the semiclassical approximation $[62,63]$,

$$
S^{\mathrm{BH}}=\frac{A}{4 l_{p}^{2}},
$$

is modified by the corresponding quantum corrections ongoing from HUP to GUP [64-67].

In particular, [65],

$$
S_{\mathrm{GUP}}^{\mathrm{BH}}=\frac{A}{4 l_{p}^{2}}-\frac{\pi \alpha^{\prime 2}}{4} \ln \left(\frac{A}{4 l_{p}^{2}}\right)+\sum_{n=1}^{\infty} c_{n}\left(\frac{A}{4 l_{p}^{2}}\right)^{-n}+\text { const., }
$$

where the expansion coefficients $c_{n} \propto \alpha^{\prime 2(n+1)}$ can always be computed to any desired order of accuracy.

The general form of quantum corrections for the black hole entropy derived in (5) remains valid for any horizon spaces and, in particular, for the holographic screen $\delta$ from [14]. Specifically, in $[68,69]$ the logarithmic correction was obtained in the following form:

$$
S_{\ln }=\frac{A}{4 l_{p}^{2}}+\frac{\widetilde{\alpha}}{4} \ln \left(\frac{A}{l_{p}^{2}}\right)
$$

omitting the Boltzmann constant $k_{B}$ as a factor and assuming it to be equal to unity in what follows. Higher-order corrections may be derived using the Taylor-series expansion in terms of the small parameter $l_{p}^{2} / A$ :

$$
S_{\mathrm{GUP}}=\frac{A}{4 l_{p}^{2}}+\frac{\tilde{\alpha}}{4} \ln \left(\frac{A}{l_{p}^{2}}\right)+\sum_{n=1}^{\infty} \widetilde{c}_{n}\left(\frac{A}{l_{p}^{2}}\right)^{-n}+\text { const. }
$$

in a similar way to the Taylor-series expansion of the righthand side in (9) in terms of the small parameter $4 l_{p}^{2} / A$. This is valid as GUP gives the ultraviolet cutoff at the level of $l_{\min } \sim$ $l_{p}$.

In this way, at high energies we have $S \rightarrow S_{\mathrm{GUP}}$, and hence $N \rightarrow N_{\mathrm{GUP}}$. Assuming in the notation of [14] that

$$
S=\frac{1}{4} N
$$

we directly obtain

$$
N_{\mathrm{GUP}}=\frac{A}{l_{p}^{2}}+\widetilde{\alpha} \ln \left(\frac{A}{l_{p}^{2}}\right)+4 \sum_{n=1}^{\infty} \widetilde{c}_{n}\left(\frac{A}{l_{p}^{2}}\right)^{-n}+\text { const. }
$$

Now, coming back to [14], in terms of $N_{\text {GUP }}$ we can define the holographic screen area, as measured at high energies, by

$$
A_{\mathrm{GUP}} \equiv G \hbar N_{\mathrm{GUP}}
$$

where $G$ and $\hbar$ are gravitational and Planck constants, respectively, and $N_{\text {GUP }}$ is given by (9). Considering that we, similar to [14], assume that the speed of light $c=1$, then, according to $l_{p}^{2}=G \hbar$, from (9) and (10), we have

$$
A_{\mathrm{GUP}}=A+G \hbar \tilde{\alpha} \ln \left(\frac{A}{G \hbar}\right)+4 G \hbar \sum_{n=1}^{\infty} \widetilde{c}_{n}\left(\frac{A}{G \hbar}\right)^{-n}+\text { const. }
$$

In this case, an exact value of the constant in the right-hand side of (11) is of no great importance, as further we need the relation (1) being primarily interested in $d A_{\mathrm{GUP}}$ rather than in $A_{\mathrm{GUP}}$; that is, the constant in the right-hand side of (11) is insignificant. So, (1) has a fairly definite analog at high energies

$$
d N_{\mathrm{GUP}}=\frac{d A_{\mathrm{GUP}}}{G \hbar}
$$

that ongoing to the known low energies gives (1). There is a single considerable difference; in [14] the quantity $N$ was defined in terms of $A$, and $d N$ was defined in terms of $d A$, but in the case under study, the situation is opposite: $A_{\mathrm{GUP}}$ is defined in terms of $N_{\mathrm{GUP}}$ and $d A_{\mathrm{GUP}}$ in terms of $d N_{\mathrm{GUP}}$. The logic series is here as follows:

$$
A \Longrightarrow N \Longrightarrow N_{\mathrm{GUP}} \Longrightarrow A_{\mathrm{GUP}} \text {. }
$$

The GUP-correction problem of the temperature $T$ for the arbitrary holographic screen $\mathcal{S}$ has been studied in [70]. Actually, this case is identical to the case of the Schwarzschild black hole, and $T_{\mathrm{GUP}}$ was derived as a series (formulas (1), (7) from [70]):

$$
T_{\mathrm{GUP}}=T\left(1+\Theta_{T} T^{2}+\cdots\right)=T+\Theta_{T} T^{3}+\cdots=T+\widetilde{T}_{\mathrm{GUP}},
$$

where the factors in the right-hand side (14) may be computed in the explicit form and at low energies $\widetilde{T}_{\mathrm{GUP}} \rightarrow 0$.

Thus, we can have a GUP - analog of Komar's mass in ((5.33) from [14])

$$
\begin{aligned}
M_{\mathrm{GUP}} & =\frac{1}{2} \int_{\mathcal{S}} T_{\mathrm{GUP}} d N_{\mathrm{GUP}} \\
& =\frac{1}{2} \int_{\mathcal{S}}\left(T+\widetilde{T}_{\mathrm{GUP}}\right) d N_{\mathrm{GUP}}=\frac{1}{2 G \hbar} \int_{\mathcal{S}} T_{\mathrm{GUP}} d A_{\mathrm{GUP}}
\end{aligned}
$$


that the low-energy limit gives the well-known Komar formula [71], [72, page 289].

It is clear that the "GUP-deformed Komar's mass" $M_{\mathrm{GUP}}$ in the first term (15) as a component has the known Komar's mass [71], [72, (11.2.9-11.2.10)], [14, (5.34)]

$$
M=\frac{1}{4 \pi G} \int_{\mathcal{S}} T d A
$$

\section{3. $N_{\mathrm{GUP}}, A_{\mathrm{GUP}}$, and $M_{\mathrm{GUP}}$ in Terms of Unified Small Parameter}

If feasible, it is desirable to express all the above-derived fundamental quantities in terms of a unified parameter. As shown by the author in $[39,73]$, this is possible for black holes within the scope of GUP, and a role of the unified small parameter is played by the parameter introduced previously in [27-36] as follows:

$$
\alpha_{x}=\frac{l_{\min }^{2}}{x^{2}}
$$

where $x$ is the measuring scale, $l_{\min } \sim l_{p}$ by virtue of GUP (3), and $0<\alpha \leq 1 / 4$.

Obviously, the principal results obtained in $[39,73]$ remain in force for an arbitrary screen $\delta$ and may be applied to the quantities $N_{\mathrm{GUP}}, A_{\mathrm{GUP}}$, and $M_{\mathrm{GUP}}$ defined in the preceding section.

Substituting from GUP (3) $l_{\text {min }}=2 \sqrt{ } \alpha^{\prime} l_{p}$ and using the formula $A=4 \pi R^{2}$, where $R$ is the radius of the screen $\mathcal{S}$, we get $N_{\mathrm{GUP}}(9)$ of the following form:

$$
N_{\mathrm{GUP}}=N+\widetilde{\alpha} \ln \left(\sigma \alpha_{R}^{-1}\right)+4 \sum_{n=1}^{\infty} \widetilde{c}_{n} \sigma^{-n} \alpha_{R}^{n}+\text { const. }
$$

Here, $\alpha_{R}$ is a value of $\alpha$ parameter at the point $R$, and $\sigma$ is equal to $16 \alpha^{\prime} \pi$. It is convenient to refer to the form $N_{\mathrm{GUP}}$ derived in (18) as to the $\alpha$-representation.

Using (10) and (11), we can easily obtain $\alpha$-representation for $A_{\mathrm{GUP}}$ :

$$
A_{\mathrm{GUP}}=A+\widetilde{\alpha} G \hbar \ln \left(\sigma \alpha_{R}^{-1}\right)+4 G \hbar \sum_{n=1}^{\infty} \widetilde{c}_{n} \sigma^{-n} \alpha_{R}^{n}+\text { const. }
$$

Also, it is clear that $M_{\mathrm{GUP}}$ (15) may be derived in terms of $\alpha_{R}$.

Here, $\alpha_{x}$ is considered as a deformation parameter for the Heisenberg algebra ongoing from HUP to GUP. Generally speaking, initially the construction of such a deformation was realized with other parameters (e.g., $[60,61]$ ). But it is easily shown that QFT parameter of the deformations associated with GUP may be expressed in terms of the parameter $\alpha$ that has been introduced in the approach to the density matrix deformation $[38,39]$. Here the notation of [74] is used. Then [39, page 943],

$$
\begin{gathered}
{[\vec{x}, \vec{p}]=i \hbar\left(1+\beta^{2} \vec{p}^{2}+\cdots\right),} \\
\Delta x_{\min } \approx \hbar \sqrt{\beta} \sim l_{p} .
\end{gathered}
$$

In this case, from (20), (21), it follows that $\beta \sim \mathbf{1} / \mathbf{p}^{2}$, and for $x_{\min } \sim l_{p}, \beta$ corresponding to $x_{\min }$ is nothing else but

$$
\beta \sim \frac{1}{P_{p l}^{2}}
$$

where $P_{p l}$ is Planck's momentum: $P_{p l}=\hbar / l_{p}$.

In this manner, $\beta$ is varying over the following interval:

$$
\frac{\lambda}{P_{p l}^{2}} \leq \beta
$$

where $\lambda$ is a numerical factor and the second term in (20) is accurately reproduced in the momentum representation (up to the numerical factor) by $\alpha_{x}=l_{\min }^{2} / x^{2} \sim l_{p}^{2} / x^{2}=p^{2} / P_{p l}^{2}$

$$
[\vec{x}, \vec{p}]=i \hbar\left(1+\beta^{2} \vec{p}^{2}+\cdots\right)=i \hbar\left(1+a_{1} \alpha_{x}+a_{2} \alpha_{x}^{2}+\cdots\right) .
$$

In the case under study, convenience of using $\alpha_{x}$ stems from its smallness, its dimensionless character, and ability to test changes in the radius $R$ of the holographic screen $\delta$.

\section{Quantum Corrections to the Principal Result and Ultraviolet Limit}

Based on the aforesaid, we can proceed the generalization of the results from Section 5.2 of [14] and the derivation of equations for a gravitational field within the scope of GUP.

We must consider two absolutely different cases.

4.1. Quantum Corrections to the Principal Result. It is assumed that the screen radius is given by $\mathcal{S}$

$$
R \gg l_{p}
$$

In terms of the deformation parameter $\alpha_{x}$ introduced in the previous section, we have

$$
\alpha_{R} \ll \frac{1}{4}
$$

So far we are not concerned with the redefinition of the lower limit for $\alpha_{R}$. This is interesting when going to the infrared limit.

Then, the principal result from the final part of Section 5.2 in [14] remains valid owing to the replacement of $M$ (formula (5.33) from [14]) by $M_{\mathrm{GUP}}=M_{\mathrm{GUP}}\left[\alpha_{R}\right]$ (15). The " $\alpha_{R}$-complement" (i.e., the difference $\widetilde{M}\left[\alpha_{R}\right]=M_{\mathrm{GUP}}\left[\alpha_{R}\right]-$ $M)$ to $M$ will be simply a (small) quantum correction for the principal result.

In this way, because in the case of GUP-corrections the left side of formula (5.33) and hence the left side in formulas (5.34), (5.35) from [14] are dependent on $\alpha_{R}$; the right sides of the corresponding formulas are also dependent on $\alpha_{R}$, in particular the quantities $T_{a b}, R_{a b}$, and $g_{a b}$ from (5.37).

But in fact, this relation at low energies ((25) or (26)) is insignificant since $\alpha_{R}$ at low energies (as distinct from high 
Planck's energies) is varying very slowly, practically showing continuity though being discrete in character.

Indeed, as long as there is the minimal length $l_{\min } \sim l_{p}$, all the lengths measured are its multiples, and hence $\alpha_{R}$ is a discrete nonuniformly varying quantity. Then, due to ((25) or (26)), the difference between two successive values of $\alpha_{R}$ is as follows:

$$
\Delta_{\min }\left[\alpha_{R}\right]=\alpha_{R}-\alpha_{R+l_{\min }} \sim \frac{l_{\min }^{3}}{R^{3}},
$$

for $R \gg l_{p}$ or, that is the same, for $R \gg l_{\min }$ giving a value close to zero.

And assuming in this case that $\alpha_{R}$ is continuously varying from $R$ and all the quantities in Section 5.2 of [14] are also continuously dependent on $\alpha_{R}$ (26), we can write down the " $\alpha$-analog" of formula (5.37) in [14] as

$$
\begin{gathered}
2 \int_{\Sigma}\left(T_{a b}[\alpha]-\frac{1}{2} T[\alpha] g_{a b}[\alpha]\right) n^{a} \xi^{b} d V \\
=\frac{1}{4 \pi G} \int_{\Sigma} R_{a b}[\alpha] n^{a} \xi^{b} d V
\end{gathered}
$$

where the dependence of $T_{a b}[\alpha]$ and $R_{a b}[\alpha]$ on $\alpha=\alpha_{R}$ is completely determined, in accordance with $[14,72]$, by the integral $M_{\mathrm{GUP}}[\alpha](15)$.

Besides, it is assumed that $n^{a}$ and $\xi^{b}$ are dependent on $\alpha$, though the dependence is dropped.

Next, similar to [14], from (28) we can derive the $\alpha$ deformed Einstein Equations using the method from [1]. Note that both this method and its minor modification given ([14], end of Section 5.2) in this case are valid because $\alpha_{R}$ is small and continuous; the whole system is being continuously dependent on it.

Solutions of the $\alpha$-deformed Einstein Equations represent a series in $\alpha_{R}$, and for $\alpha_{R} \rightarrow 0$ or for $\alpha^{\prime}=0$ from formula (3) become the corresponding solutions of Section 5.2 in [14].

Using the result obtained in [75], we can easily extend the above result to the case with a nonzero cosmological term $\Lambda \neq 0$. In [75], Komar's formula was generalized to the case of a nonzero $\Lambda$. All the arguments from Section 5.2 of [14] in this case remain valid, and formula (5.37) takes the following form:

$$
\begin{aligned}
2 \int_{\Sigma}( & \left.T_{a b}-\frac{1}{2} T g_{a b}\right) n^{a} \xi^{b} d V \\
& =\frac{1}{4 \pi G} \int_{\Sigma}\left(R_{a b}+\Lambda g_{a b}\right) n^{a} \xi^{b} d V .
\end{aligned}
$$

We can easily obtain the $\alpha$-analog of the last formula with the dynamic cosmological term $\Lambda(\alpha)$ as a corresponding complement to the right-hand side (28). Analysis of the relationship between $\Lambda$ and $\alpha$, applicable in this case as well will be given in Section 4.2.

4.2. Ultraviolet Limit. In the case in question, we suggest that the screen $\mathcal{S}$ has a radius on the order of several Planck's lengths

$$
R \approx \xi l_{\min }=2 \alpha^{\prime} \xi l_{p}
$$

where $\xi$ is a number on the order of 1 or

$$
\alpha_{R} \approx \frac{1}{4} \text {. }
$$

The problem is which object puts the limit for such a screen $\mathcal{S}$. It may be assumed that if $T_{a b} \neq 0$ then the object may be represented only by Planck's black hole or by a microblack hole with a radius on the order of several Planck's lengths.

Clearly, the methods of $[1,14]$ are not in force for such screen $\mathcal{S}$. Specifically, there are no classical analogs of $N$, $T$, and $M$ for the screen. Moreover, it is impossible to use the result of [1] as "a very small region the space-time" is no longer "an approximate Minkowski space" [14].

Also, such microblack hole is a horizon space, jet at high energies (Planck scales). As is known, for horizon spaces, black holes in particular, at low energies (semiclassical approximation) the results of [12] are valid.

At the horizon (and we are interested in this case only) Einstein's field Equations may be written as a thermodynamic identity ([12] formula (119)):

$$
\underbrace{\frac{\hbar c f^{\prime}(a)}{4 \pi} \frac{c^{3}}{G \hbar} d\left(\frac{1}{4} 4 \pi a^{2}\right)-\frac{1}{2} \frac{c^{4} d a}{G}}_{k_{B} T}=\underbrace{P d\left(\frac{4 \pi}{3} a^{3}\right)}_{d S},
$$

where $R=a$ is a radius of a black hole (i.e., of the screen $\mathcal{S}), P=T_{R}^{R}$ is the trace of the momentum-energy tensor and radial pressure, and the horizon location will be given by simple zero of the function $f(R)$, at $R=a$.

The main ingredients of (32) may be written in terms of the deformation parameter $\alpha$ with the coefficients containing only the numerical factors and fundamental constants [73].

Also, the work [73] presents two possible variants of highenergy (Planck) $\alpha$-deformation $\alpha \rightarrow 1 / 4$ (32).

Hereinafter, we assume that the energy-momentum tensor of matter fields is not traceless:

$$
T_{a}^{a} \neq 0
$$

similar, in particular, to the case under study (32) $P=T_{R}^{R} \neq 0$.

4.2.1. Case of Equilibrium Thermodynamics [73, Section 6.1]. In this case, it is assumed that in the high-energy (ultraviolet (UV)) limit the thermodynamic identity (32) is retained but now all the quantities involved in this identity become $\alpha$ deformed ( $\alpha \rightarrow 1 / 4)$. All the quantities $\Upsilon$ in (32) are replaced by the corresponding quantities $\Upsilon_{\mathrm{GUP}}$ with the subscript GUP. Then, the high-energy $\alpha$-deformation of (32) takes the form

$$
k_{B} T_{\mathrm{GUP}}(\alpha) d S_{\mathrm{GUP}}(\alpha)-d E_{\mathrm{GUP}}(\alpha)=P(\alpha) d V_{\mathrm{GUP}}(\alpha) \text {. }
$$

Substituting into (34) the corresponding quantities $T_{\mathrm{GUP}}(\alpha)$, $S_{\mathrm{GUP}}(\alpha), E_{\mathrm{GUP}}(\alpha), V_{\mathrm{GUP}}(\alpha)$, and $P(\alpha)$ and expanding them into a Laurent series in terms of $\alpha$, close to high values of $\alpha$, specifically close to $\alpha=1 / 4$, we can derive a solution for the high energy $\alpha$-deformation of the general relativity (34) as a function of $P(\alpha)$. Provided at high energies the generalization of (32) to (34) is possible; we can have the high-energy $\alpha$ deformation of the metric. 
It is noteworthy that in (34) $T_{\mathrm{GUP}}$ this time is calculated from [64, formula (10)]

$$
\begin{aligned}
T_{\mathrm{GUP}}^{\mathrm{BH}} & =\frac{1}{4 \pi} \frac{\hbar R}{2 \alpha^{\prime 2} l_{p}^{2}}\left[1-\sqrt{1-\frac{\alpha^{\prime 2} l_{p}^{2}}{R^{2}}}\right] \\
& =\frac{\hbar \alpha_{R}^{-1}}{4 \pi \alpha^{\prime} l_{p}}\left[1-\left(1-\alpha_{R}\right)^{1 / 2}\right]
\end{aligned}
$$

with subsequent replacement of $l_{p}$ by $\sqrt{G \hbar}$ for $c=1$.

It is especially interesting to consider the following case.

4.2.2. Case of Nonequilibrium Thermodynamics [73, Section 6.2]. In this case, the $\alpha$-dependent dynamic cosmological term $\Lambda(\alpha) \neq 0$ appears in the right-hand side of (34). Then, with the addition of $\Lambda(\alpha) \neq 0$, the $\alpha$-representation (34) (for $\hbar=1)$ is given as follows [73, formula (53)]:

$$
-\alpha^{2} f^{\prime}(\alpha)-\frac{1}{2} \alpha=16 \pi \alpha^{\prime 2} P(\alpha) G^{2}-G \Lambda(\alpha),
$$

where $\alpha=\alpha_{R} \approx 1 / 4$,

$$
f^{\prime}(\alpha)=4 \pi k_{B} T_{\mathrm{GUP}}(\alpha)
$$

and the derivative in the left-hand side of (37) is taken with respect to $\alpha$.

$\Lambda(\alpha)$ in the right-hand side of (37) may be subjected to a series expansion in terms of $\alpha$, in compliance with the holographic principle [49-53] as applied to the Universe [76]. In $[38,39,73,77]$ in the leading order, this expansion results in the first power, that is, we have

$$
\Lambda\left(\alpha_{R}\right) \sim \alpha_{R} \Lambda_{p}
$$

where $\Lambda_{p}$ is the initial value of $\Lambda \approx \Lambda_{1 / 4}$ derived using the well-known procedure of "summation over all zero modes" and the Planck momentum cutoff $[78,79]$. Actually, $(38)$ is in a good agreement with the observable $\Lambda=\Lambda_{\text {observ }}$. Because a radius of the visible part of the Universe is given as $R=$ $R_{\text {Univ }} \approx 10^{28} \mathrm{~cm}$, it is clear that $\alpha_{R} \approx 10^{-122}$, and (38) is completely consistent with the experiment [79].

Note that proceeding directly from a quantum field theory but without the use of the holographic principle, we can have only a rough estimate of $\Lambda$ that, on the whole, is at variance with $\Lambda_{\text {observ }}$. Such an estimate may be obtained in different ways: by simulation [80]; using the cutoff [78] but now in the infrared limit; with the use of the Generalized Uncertainty Principle for the pair $(\Lambda, V)$, where $V$ is fourdimensional volume [38,39]. In the $\alpha$-representation in this case, the expansion in terms of $\alpha$ results in the second leading order

$$
\Lambda\left(\alpha_{R}\right) \sim \alpha_{R}^{2} \Lambda_{p}
$$

that, obviously, is at variance with the accepted facts.

\section{Conclusion}

(I) A very interesting case of the zero energy-momentum tensor for matter fields $T_{a b}=0$ and, specifically the case of $P(\alpha)=0$ in the right-hand side of (36) has remained beyond the scope of the final section. We can state the problem more specifically: for which conditions in this case we can derive a solution in the form of the de Sitter space with large values of $\alpha$ ?

This problem is also important when we try to find whether it is possible to derive the initial inflation conditions $[81,82]$ for $T_{a b}=0$ on the basis of the foregoing analysis.

Note that the dynamic cosmological term $\Lambda(\alpha)$ correlates well with inflation models $[81,82]$ as the latter require a very high $\Lambda$ at the early stages of the Universe, and this is distinct from $\Lambda=\Lambda_{\text {exper }}$ in the modern period. Of great interest is the recent work [83], where a mechanism of the vacuum energy decay in the de Sitter space is established to support a dynamic nature of $\Lambda$.

(II) The deformation parameter $\alpha_{R}$ has a double meaning.

As $\kappa=1 / R$-curvature with the radius $R, \alpha_{R}=\kappa^{2} l_{\min }^{2}$ is nothing else but the squared curvature multiplied by the squared minimal area and is explicitly dependent on the energy $E$. On the other hand, it is seen that, at least at the known energies, from the definition of the bit number $N$ in $\left[14\right.$, formula (3.10)], we get $\alpha_{R} \sim 1 / N$. But, because $\alpha_{R}=$ $\alpha_{R}[E]$, this suggests that $N=N[E]$, as demonstrated in the text ongoing to higher energies

$$
N \Longrightarrow N_{\mathrm{GUP}} \text {. }
$$

Nevertheless, ongoing to lower energies, that is, in the infrared limit, the same should be true: the bit number must be a function of energy.

This problem and the relevant questions touched upon in this paper will be further considered in subsequent works of the author.

\section{References}

[1] T. Jacobson, "Thermodynamics of spacetime: the Einstein equation of state," Physical Review Letters, vol. 75, no. 7, pp. 1260-1263, 1995.

[2] T. Padmanabhan, "A new perspective on gravity and dynamics of space-time," International Journal of Modern Physics D, vol. 14, no. 12, pp. 2263-2270, 2005.

[3] T. Padmanabhan, "The holography of gravity encoded in a relation between entropy, horizon area, and action for gravity," General Relativity and Gravitation, vol. 34, no. 12, pp. 20292035, 2002.

[4] T. Padmanabhan, "Holographic gravity and the surface term in the Einstein-Hilbert action," Brazilian Journal of Physics, vol. 35, no. 2, pp. 362-372, 2005.

[5] T. Padmanabhan, "Gravity: a new holographic perspective," International Journal of Modern Physics D, vol. 15, no. 10, pp. 1659-1676, 2006.

[6] A. Mukhopadhyay and T. Padmanabhan, "Holography of gravitational action functionals," Physical Review D, vol. 74, no. 12, Article ID 124023, 15 pages, 2006.

[7] T. Padmanabhan, "Dark energy and gravity," General Relativity and Gravitation, vol. 40, no. 2-3, pp. 529-564, 2008. 
[8] T. Padmanabhan and A. Paranjape, "Entropy of null surfaces and dynamics of spacetime," Physical Review D, vol. 75, no. 6, Article ID 064004, 16 pages, 2007.

[9] T. Padmanabhan, "Gravity as an emergent phenomenon: a conceptual description," AIP Conference Proceedings, vol. 939, pp. 114-123, 2007.

[10] T. Padmanabhan, "Gravity and the thermodynamics of horizons," Physics Reports, vol. 406, no. 2, pp. 49-125, 2005.

[11] A. Paranjape, S. Sarkar, and T. Padmanabhan, "Thermodynamic route to field equations in Lanczos-Lovelock gravity," Physical Review D, vol. 74, no. 10, Article ID 104015, 9 pages, 2006.

[12] T. Padmanabhan, "Thermodynamical aspects of gravity: new insights," Reports on Progress in Physics, vol. 73, Article ID 046901, 2010.

[13] T. Padmanabhan, "Equipartition of energy in the horizon degrees of freedom and the emergence of gravity," Modern Physics Letters A, vol. 25, no. 14, pp. 1129-1136, 2010.

[14] E. Verlinde, "On the origin of gravity and the laws of Newton," Journal of High Energy Physics, vol. 2011, no. 4, article 29, 2011.

[15] D. A. Easson, P. H. Frampton, and G. F. Smoot, "Entropic accelerating universe," Physics Letters B, vol. 696, no. 3, pp. 273277, 2011.

[16] R. A. Konoplya, "Entropic force, holography and thermodynamics for static space-times," European Physical Journal C, vol. 69, no. 3, pp. 555-562, 2010.

[17] P. Nicolini, "Entropic force, noncommutative gravity, and ungravity," Physical Review D, vol. 82, no. 4, Article ID 044030, 8 pages, 2010.

[18] C. Gao, "Modified entropic force," Physical Review D, vol. 81, no. 8, Article ID 087306, 4 pages, 2010.

[19] Y. S. Myung and Y. W. Kim, "Entropic force and entanglement system," Physical Review D, vol. 81, no. 10, Article ID 105012, 7 pages, 2010.

[20] R. Banerjee and B. R. Majhi, "Statistical origin of gravity," Physical Review D, vol. 81, no. 12, Article ID 124006, 5 pages, 2010.

[21] F. Caravelli and L. Modesto, "Holographic effective actions from black holes," Physics Letters B, vol. 702, no. 4, pp. 307-311, 2011.

[22] L. Modesto and A. Randono, "Entropic corrections to Newton's law," http://arxiv.org/abs/1003.1998.

[23] C. M. Ho, D. Minic, and Y. J. Ng, "Cold dark matter with MOND scaling,” Physics Letters B, vol. 693, no. 5, pp. 567-570, 2010.

[24] J. R. Mureika and R. B. Mann, "Does entropic gravity bound the masses of the photon and graviton?" Modern Physics Letters A, vol. 26, no. 3, pp. 171-181, 2011.

[25] V. V. Kiselev and S. A. Timofeev, "The holographic screen at low temperatures," Modern Physics Letters A, vol. 26, no. 2, pp. 109118, 2011.

[26] H. Sahlmann, "Newton's constant from a minimal length: additional models," Classical and Quantum Gravity, vol. 28, no. 1, Article ID 015006, 6 pages, 2011.

[27] A. E. Shalyt-Margolin and J. G. Suarez, "Quantum mechanics of the early universe and its limiting transition," http://arxiv.org/ abs/gr-qc/0302119.

[28] A. E. Shalyt-Margolin and J. G. Suarez, "Quantum mechanics at Planck's scale and density matrix," International Journal of Modern Physics D, vol. 12, no. 7, pp. 1265-1278, 2003.

[29] A. E. Shalyt-Margolin and A. Y. Tregubovich, "Deformed density matrix and generalized uncertainty relation in thermodynamics," Modern Physics Letters A, vol. 19, no. 1, pp. 71-82, 2004.
[30] A. E. Shalyt-Margolin, "Non-unitary and unitary transitions in generalized quantum mechanics, new small parameter and information problem solving," Modern Physics Letters A, vol. 19, no. 5, pp. 391-403, 2004.

[31] A. E. Shalyt-Margolin, "Pure states, mixed states and Hawking problem in generalized quantum mechanics," Modern Physics Letters A, vol. 19, no. 27, pp. 2037-2045, 2004.

[32] A. E. Shalyt-Margolin, "The universe as a nonuniform lattice in finite-volume hypercube: I. Fundamental definitions and particular features," International Journal of Modern Physics D, vol. 13, no. 5, pp. 853-864, 2004.

[33] A. E. Shalyt-Margolin, "The Universe as a nonuniform lattice in the finite-dimensional hypercube. II. Simple cases of symmetry breakdown and restoration," International Journal of Modern Physics A, vol. 20, no. 20-21, pp. 4951-4964, 2005.

[34] A. E. Shalyt-Margolin and V. I. Strazhev, "The density matrix deformation in quantum and statistical mechanics of the early universe," in Proceedings of the 6th International Symposium "Frontiers of Fundamental and Computational Physics", B. G. Sidharth, Ed., pp. 131-134, Springer, Berlin, Germany, 2006.

[35] A. E. Shalyt-Margolin, "The density matrix deformation in physics of the early universe and some of its implications," in Quantum Cosmology Research Trends, A. Reimer, Ed., Horizons in World Physics no. 246, pp. 49-92, Nova Science, Hauppauge, NY, USA, 2005.

[36] A. E. Shalyt-Margolin, "Deformed density matrix and quantum entropy of the black hole," Entropy, vol. 8, no. 1, pp. 31-43, 2006.

[37] A. E. Shalyt-Margolin, "Entropy in the present and early Universe," Symmetry, vol. 18, pp. 299-320, 2007.

[38] A. E. Shalyt-Margolin, "Entropy in the present and early universe and vacuum energy," AIP Conference Proceedings, vol. 1205, pp. 160-167, 2010.

[39] A. Shalyt-Margolin, "Entropy in the present and early universe: new small parameters and dark energy problem," Entropy, vol. 12, no. 4, pp. 932-952, 2010.

[40] L. Garay, "Quantum gravity and minimum length," International Journal of Modern Physics A, vol. 10, no. 2, pp. 145-146, 1995.

[41] F. Scardigli, "Generalized uncertainty principle in quantum gravity from micro-black hole gedanken experiment," Physics Letters B, vol. 452, no. 1-2, pp. 39-44, 1999.

[42] M. Maggiore, "A generalized uncertainty principle in quantum gravity," Physics Letters B, vol. 304, no. 1-2, pp. 65-69, 1993.

[43] A. Ashtekar and J. Lewandowski, "Background independent quantum gravity: a status report," Classical and Quantum Gravity, vol. 21, no. 15, pp. R53-R152, 2004.

[44] C. Kiefer, "Quantum gravity: general introduction and recent developments," Annalen der Physik, vol. 15, no. 1-2, pp. 129-148, 2006.

[45] M. Han, W. Huang, and Y. Ma, "Fundamental structure of loop quantum gravity," International Journal of Modern Physics D, vol. 16, no. 9, pp. 1397-1474, 2007.

[46] V. A. Rubakov and P. G. Tinyakov, "Infrared-modified gravities and massive gravitons," Physics Uspekhi, vol. 51, no. 8, pp. 759792, 2008.

[47] V. Nikiforova, S. Randjbar-Daemi, and V. Rubakov, "Infrared modified gravity with dynamical torsion," Physical Review D, vol. 80, no. 12, Article ID 124050, 10 pages, 2009.

[48] S. G. Turyshev, "Experimental tests of general relativity: recent progress and future directions," Physics Uspekhi, vol. 52, no. 1, pp. 1-27, 2009. 
[49] G. 't Hooft, "Dimensional reduction in quantum gravity," http:// arxiv.org/abs/gr-qc/9310026.

[50] G. 't Hooft, “The holographic principle," http://arxiv.org/abs/ hep-th/0003004.

[51] L. Susskind, “The world as a hologram," Journal of Mathematical Physics, vol. 36, no. 11, pp. 6377-6396, 1995.

[52] R. Bousso, “The holographic principle," Reviews of Modern Physics, vol. 74, no. 3, pp. 825-874, 2002.

[53] R. Bousso, "A covariant entropy conjecture," Journal of High Energy Physics, vol. 1999, no. 7, article 4, 1999.

[54] G. A. Veneziano, "Stringy nature needs just two constants," Europhysics Letters, vol. 2, no. 3, pp. 199-211, 1986.

[55] D. Amati, M. Ciafaloni, and G. Veneziano, "Can spacetime be probed below the string size?” Physics Letters B, vol. 216, no. 1-2, pp. 41-47, 1989.

[56] E. Witten, "Reflections on the fate of spacetime," Physics Today, vol. 49, no. 4, pp. 24-28, 1996.

[57] R. J. Adler and D. I. Santiago, "On gravity and the uncertainty principle," Modern Physics Letters A, vol. 14, no. 20, pp. 13711378, 1999.

[58] D. V. Ahluwalia, "Wave-particle duality at the Planck scale: freezing of neutrino oscillations," Physics Letters A, vol. 275, no. 1-2, pp. 31-35, 2000.

[59] D. V. Ahluwalia, "Interface of gravitational and quantum realms," Modern Physics Letters A, vol. 17, no. 15-17, pp. 11351145, 2002.

[60] M. Maggiore, "The algebraic structure of the generalized uncertainty principle," Physics Letters B, vol. 319, no. 1-3, pp. 83-86, 1993.

[61] A. Kempf, G. Mangano, and R. B. Mann, "Hilbert space representation of the minimal length uncertainty relation," Physical Review D, vol. 52, no. 2, pp. 1108-1118, 1995.

[62] J. D. Bekenstein, "Black holes and entropy", Physical Review D, vol. 7, pp. 2333-2346, 1973.

[63] S. W. Hawking, "Black holes and thermodynamics," Physical Review D, vol. 13, no. 2, pp. 191-197, 1976.

[64] M. I. Park, “The generalized uncertainty principle in (A)dS space and the modification of Hawking temperature from the minimal length," Physics Letters B, vol. 659, no. 3, pp. 698-702, 2008.

[65] A. J. M. Medved and E. C. Vagenas, "When conceptual worlds collide: the generalized uncertainty principle and the Bekenstein-Hawking entropy," Physical Review D, vol. 70, no. 12, Article ID 124021, 5 pages, 2004.

[66] W. Kim, E. J. Son, and M. Yoon, "Thermodynamics of a black hole based on a generalized uncertainty principle," Journal of High Energy Physics, vol. 2008, no. 1, article 35, 2008.

[67] K. Nouicer, "Quantum-corrected black hole thermodynamics to all orders in the Planck length," Physics Letters B, vol. 646, no. 2-3, pp. 63-71, 2007.

[68] Y. Zhang, Y. G. Gong, and Z. H. Zhu, "Modified gravity emerging from thermodynamics and holographic principle," International Journal of Modern Physics D, vol. 20, no. 8, pp. 1505-1519, 2011.

[69] R. G. Cai, L. M. Cao, and Y. P. Hu, "Corrected entropy-area relation and modified Friedmann equations," Journal of High Energy Physics, vol. 2008, no. 8, article 90, 2008.

[70] M. R. Setare, D. Momeni, and R. Myrzakulov, "Entropic corrections to Newton's law," Physica Scripta, vol. 85, no. 6, Article ID 065007, 2012.
[71] A. Komar, "Covariant conservation laws in general relativity," Physical Review, vol. 113, pp. 934-936, 1959.

[72] R. M. Wald, General Relativity, University of Chicago Press, Chicago, Ill, USA, 1984.

[73] A. E. Shalyt-Margolin, "Quantum theory at planck scale, limiting values, deformed gravity and dark energy problem," International Journal of Modern Physics D, vol. 21, no. 2, Article ID 1250013, 20 pages, 2012.

[74] Y. W. Kim, H. W. Lee, and Y. S. Myung, "Entropy bound of local quantum field theory with generalized uncertainty principle," Physics Letters B, vol. 673, no. 4-5, pp. 293-296, 2009.

[75] A. Magnon, "On Komar integrals in asymptotically anti de Sitter space-times," Journal of Mathematical Physics, vol. 26, no. 12, pp. 3112-3117, 1985.

[76] W. Fischler and L. Susskind, "Holography and cosmology," http://arxiv.org/abs/hep-th/9806039.

[77] C. Balazs and I. Szapudi, "Naturalness of the vacuum energy in holographic theories," http://arxiv.org/abs/hep-th/0603133.

[78] Y. B. Zel'dovich, "The cosmological constant and the theory of elementary particles," Soviet Physics Uspekhi, vol. 11, no. 3, pp. 381-393, 1968.

[79] S. Weinberg, “The cosmological constant problem," Reviews of Modern Physics, vol. 61, no. 1, pp. 1-23, 1989.

[80] M. A. Markov, "Ultimate matter density as the universal low of nature," Pis'ma v ZHETF, vol. 36, pp. 214-216, 1982.

[81] E. W. Kolb and M. S. Turner, The Early Universe, vol. 69 of Frontiers in Physics, Addison-Wesley, Reading, Mass, USA, 1990.

[82] D. Baumann, “TASI lectures on ination,” http://arxiv.org/abs/ 0907.5424 .

[83] A. M. Polyakov, "Decay of vacuum energy," Nuclear Physics B, vol. 834, no. 1-2, pp. 316-329, 2010. 

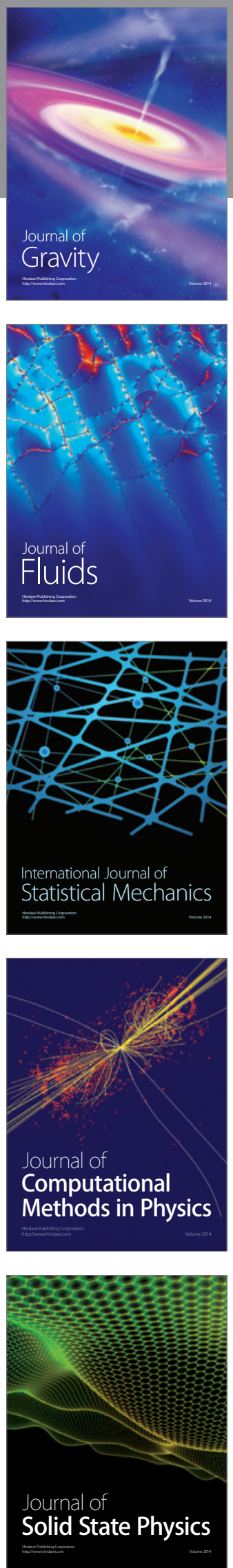

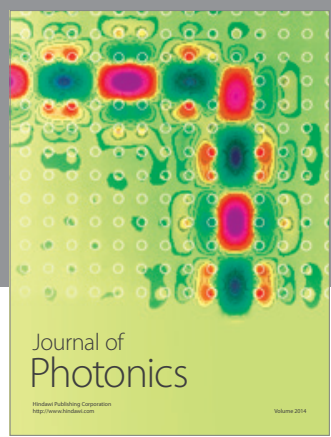

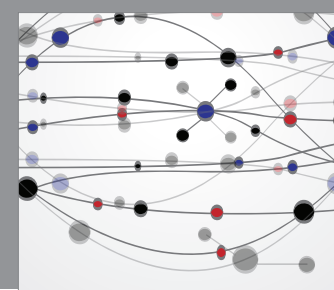

The Scientific World Journal

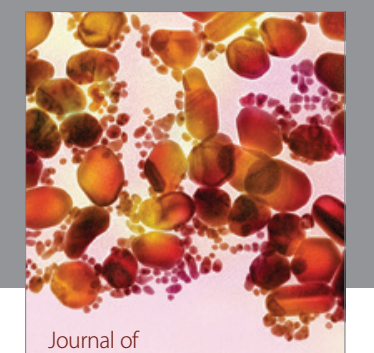

Soft Matter
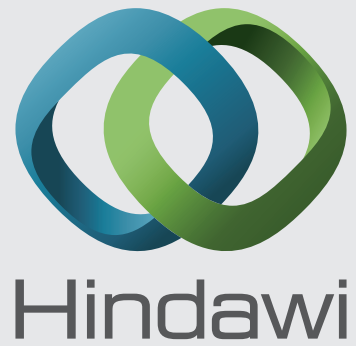

Submit your manuscripts at

http://www.hindawi.com
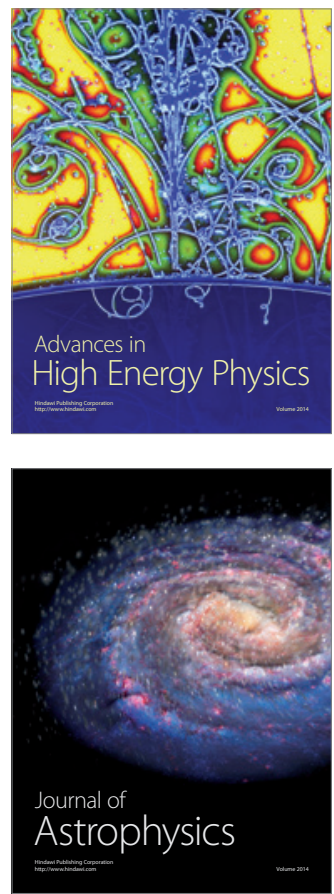
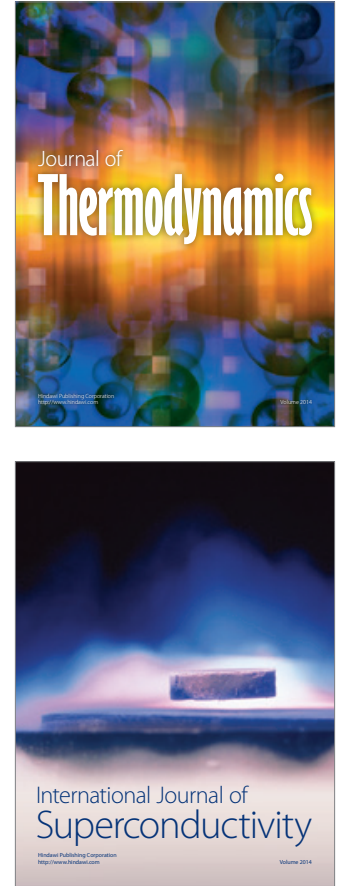
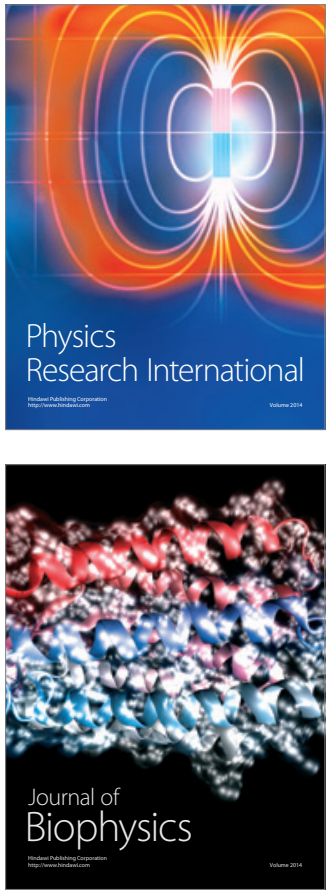
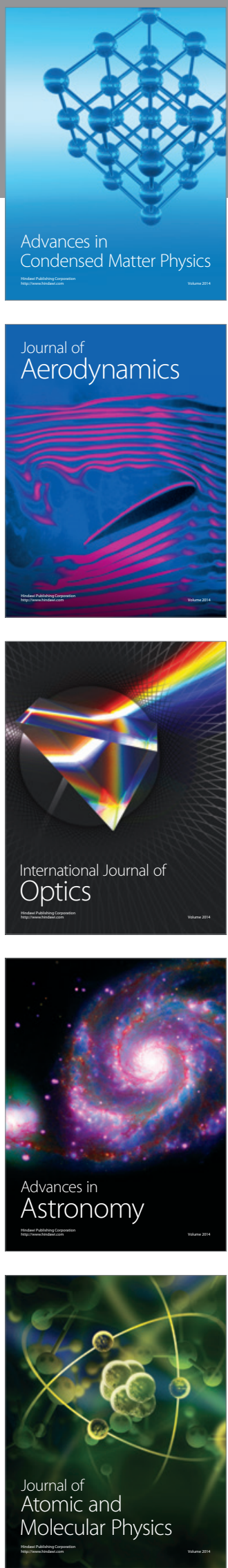